\title{
Research on Quantification of Electric Energy Loss and Treatment Scheme for Three-phase Imbalance of Distribution Network
}

\author{
Haifeng Huang ${ }^{1, a}$, Wei $\mathrm{Fu}^{1, \mathrm{~b}}$, Jun Wang ${ }^{1, \mathrm{c}}$,Chupeng Xiao ${ }^{2, \mathrm{a}}$ \\ ${ }^{1}$ State grid Hubei energy service Co.,Ltd., Wuhan,430077, China; \\ ${ }^{2}$ Nari(Wuhan) electrical equipment \&engineering efficiency evaluation center, Wuhan 430074 , \\ China. \\ a181259409@qq.com, b283727819@qq.com, c196888590@qq.com
}

Keywords: Three-phase imbalance, energy saving, treatment scheme, area network.

\begin{abstract}
Three-phase imbalance is one of the power quality problems which is more attention for distribution network especially rural distribution network. In this paper, the power loss due to three-phase imbalance was studied, and the loss quantification method of three-phase imbalance was deduced, the energy saving calculation method after the three phase unbalanced governance was put forward, the treatment scheme for unbalanced three-phase distribution network was given out, and has been carried on the practical application, provided the beneficial reference for three-phase unbalanced distribution network management and economic accounting.
\end{abstract}

\section{Introduction}

With the development of electric power system, the phenomenon of three-phase imbalance is increasingly prominent for distribution network especially the rural distribution network. When three-phase asymmetric load exists, in addition to lead to a rotary motor rotor heat damage, relay protection misoperation, heavy load phase equipment overload, will increase the extra power loss for distribution network ,sometimes, the increased loss may be many times of the loss of three-phase symmetrical distributed load.

In the usual line loss calculation method of distribution network, has no regard for unbalanced operation mostly, this has brought certain influence for the energy conservation and loss reduction of power distribution network, which could not quantize energy saving efficiency of distribution network loss reduction transformation comprehensively. This paper derives the quantitative formula of loss of the transformer and line caused by three-phase imbalance, puts forward the treatment scheme for unbalanced three-phase distribution network which could be applied to rural power distribution network, and certify the energy saving after the governance for three-phase imbalance, confirmed the feasibility of loss quantification method for three-phase imbalance.

\section{The quantitative analysis of energy loss caused by three-phase imbalance}

When three-phase load of distribution network is unbalanced, each phase of the load current is not equal, the unbalanced current will produce loss in phase line, but also cause extra loss in the neutral line $^{[2]}$, increase the total line loss, also cause additional power loss of transformer.

\subsection{Line loss caused by three-phase imbalance}

The general definition of current unbalanced degree is that:

$$
\beta=\frac{I_{\max \cdot \Phi}-I_{a v}}{I_{a v}} \times 100 \%
$$

In the formula:

$I_{\max \cdot \Phi}$ the maximum phase load current;

$I_{a v} \longrightarrow$ the average load current, $I_{a v}=\left(I_{a}+I_{b}+I_{c}\right) / 3$

Introduce the concept of phase degree of unbalance 


$$
\beta_{\Phi}=\frac{I_{\Phi}-I_{a v}}{I_{a v}} \times 100 \%
$$

In the formula:

\section{$I_{\Phi} \_$phase current;}

Obviously, $I_{\Phi}=\left(1+\beta_{\Phi}\right) I_{a v}, \quad \beta_{a}+\beta_{b}+\beta_{c}=0$, assume that phase line and neutral line resistance of three-phase four-wire system is $\mathrm{R}$, the power factor is same, when three phase is unbalanced, the loss of phase is:

$$
\Delta P_{\Phi}=\left[\left(1+\beta_{a}\right)^{2}+\left(1+\beta_{b}\right)^{2}+\left(1+\beta_{c}\right)^{2}\right] \cdot I_{a v}^{2} R
$$

Simplify:

$$
\Delta P_{\Phi}=\left[3+\beta_{a}{ }^{2}+\beta_{b}{ }^{2}+\beta_{c}{ }^{2}\right] \cdot I_{a v}^{2} R,
$$

The current of neutral is:

Follow as,

$$
\dot{I}_{N}=\left(1+\beta_{a}\right) I_{a v}+\left(1+\beta_{b}\right)\left(-\frac{1}{2}-j \frac{\sqrt{3}}{2}\right) I_{a v}+\left(1+\beta_{c}\right)\left(-\frac{1}{2}+j \frac{\sqrt{3}}{2}\right) I_{a v},
$$

$$
I_{N}^{2}=\left[\left(\beta_{a}-\frac{1}{2} \beta_{b}-\frac{1}{2} \beta_{c}\right)^{2}+\left(\frac{\sqrt{3}}{2} \beta_{c}-\frac{\sqrt{3}}{2} \beta_{b}\right)^{2}\right] I_{a v}^{2}
$$

Clear up,

$$
I_{N}^{2}=\left[\beta_{a}^{2}+\beta_{b}^{2}+\beta_{c}^{2}-\beta_{a} \beta_{b}-\beta_{b} \beta_{c}-\beta_{c} \beta_{a}\right] \cdot I_{a v}^{2} ;
$$

The power loss of neutral line is:

$$
\Delta P_{N}=I_{N}^{2} R
$$

So the total loss is:

$$
\Delta P_{u n b}=\Delta P_{\Phi}+\Delta P_{N}=\left[3+2\left(\beta_{a}^{2}+\beta_{b}{ }^{2}+\beta_{c}^{2}\right)-\left(\beta_{a} \beta_{b}+\beta_{b} \beta_{c}+\beta_{c} \beta_{a}\right)\right] \cdot I_{a v}^{2} R
$$

Define the line loss rate of increase caused by three-phase imbalance:

$r=\frac{\Delta P_{u n b}-\Delta P_{b}}{\Delta P_{b}} \times 100 \%$

Then,

$$
r=\left[2\left(\beta_{a}^{2}+\beta_{b}{ }^{2}+\beta_{c}^{2}\right)-\left(\beta_{a} \beta_{b}+\beta_{b} \beta_{c}+\beta_{c} \beta_{a}\right)\right] / 3
$$

Take $\beta_{c}=-\beta_{a}-\beta_{b}$ to simplify:

Compared the two type:

$$
\begin{gathered}
r=5\left(\beta_{a}^{2}+\beta_{b}{ }^{2}+\beta_{a} \beta_{b}\right) / 3 \\
\Delta P_{u n b}=(r+1) \Delta P_{b}, \\
\Delta E_{u n b}=(r+1) \Delta E_{b}, \\
\Delta E_{u n b}^{\prime}=\left(r^{\prime}+1\right) \Delta E_{b}
\end{gathered}
$$

$$
\frac{\Delta E_{u n b}^{\prime}}{\Delta E_{u n b}}=\frac{r^{\prime}+1}{r+1}
$$

The energy-saving after the three-phase imbalance governance $\Delta(\Delta E)$ :

$$
\begin{gathered}
\Delta(\Delta E)=\Delta E_{\text {unb }}-\Delta E_{\text {unb }}^{\prime} \\
\Delta(\Delta E)=\Delta E_{\text {unb }} \frac{r-r^{\prime}}{r+1}
\end{gathered}
$$




\subsection{The transformer loss caused by three-phase imbalance}

There are two main kinds of power loss for distribution transformer, one is no-load loss (iron loss); another is short-circuit loss (copper loss). Normally, no-load loss is fixed. Due to the load of transformer is changing, the copper loss is also changing, in the case of ignoring other factors, there is square relation between load loss and the load current in the transformer. When the Operation of the distribution transformer is unbalanced, total loss of the three-phase winding can be calculated as:

$$
P_{C u 1}=\left(I_{a}^{2}+I_{b}^{2}+I_{c}^{2}\right) R_{f}=\left(3+\beta_{a}^{2}+\beta_{b}^{2}+\beta_{c}^{2}\right) I_{a v}^{2} R_{f}
$$

In the formula, $R_{f}$ is the secondary winding resistance three-phase transformer;

When the three-phase load is balanced, every phase winding current is equal, the total loss of the three-phase winding is:

$$
P_{\text {Cu2 }}=3\left[\left(I_{a}+I_{b}+I_{c}\right) / 3\right]^{2} R_{f}
$$

The additional loss caused by the unbalanced three-phase transformer is:

$$
\Delta P=P_{\mathrm{Cu} 1}-P_{\mathrm{Cu} 2}
$$

\section{3. the management technology of three-phase imbalance for distribution network}

So far, there are three-phase reactive power compensator, three-phase automatic phase switcher, artificial adjustment phase to govern the three-phase imbalance of distribution network.

\subsection{The TSC type reactive power compensator}

TSC type dynamic reactive power compensation device mainly consists of control system and TSC main circuit. When the the high-voltage distribution network exists serious unbalanced three-phase, TSC type compensation device can be used to compensate the system its compensation principle follows as:

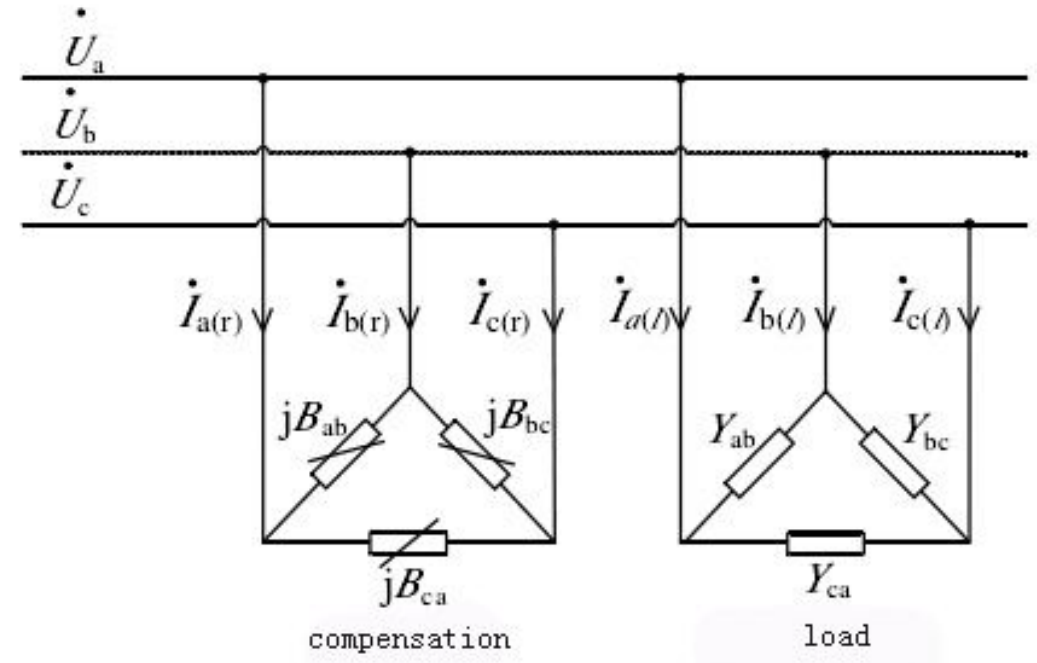

Figure 1 Three-phase unbalanced load TSC reactive compensation principle

In a three-phase system, the inductor and capacitor which jumper the phase line has the function to transfer the active power, by controlling the size of inductance and capacitance, to compensate the negative sequence and zero sequence current, achieve the goal of governance three-phase imbalance. There are many control algorithms for TSC type reactive power compensator, most of them is based on Steinmetz equilibration principle, such as balanced compensation method based on symmetrical component principle, etc. The control strategies have nine area chart, the power factor and maintain voltage control and so on. 


\subsection{Automatic phase switcher for three-phase imbalance}

Automatic phase switcher is mainly composed by main controller and automatic switching unit, main controller used to collect three phase current and neutral line current, and calculate the degree transformer is not balance, according to the export of three phase current, neutral line current, and the nodes connected to the phase sequence and user load, through balancing control strategy, get the best phase transition. By automatically switching phase sequence, reduce the harm done by the unbalanced three phase current, make the network running smoothly, improve the quality of power supply, the compensation principle is shown in figure 2.

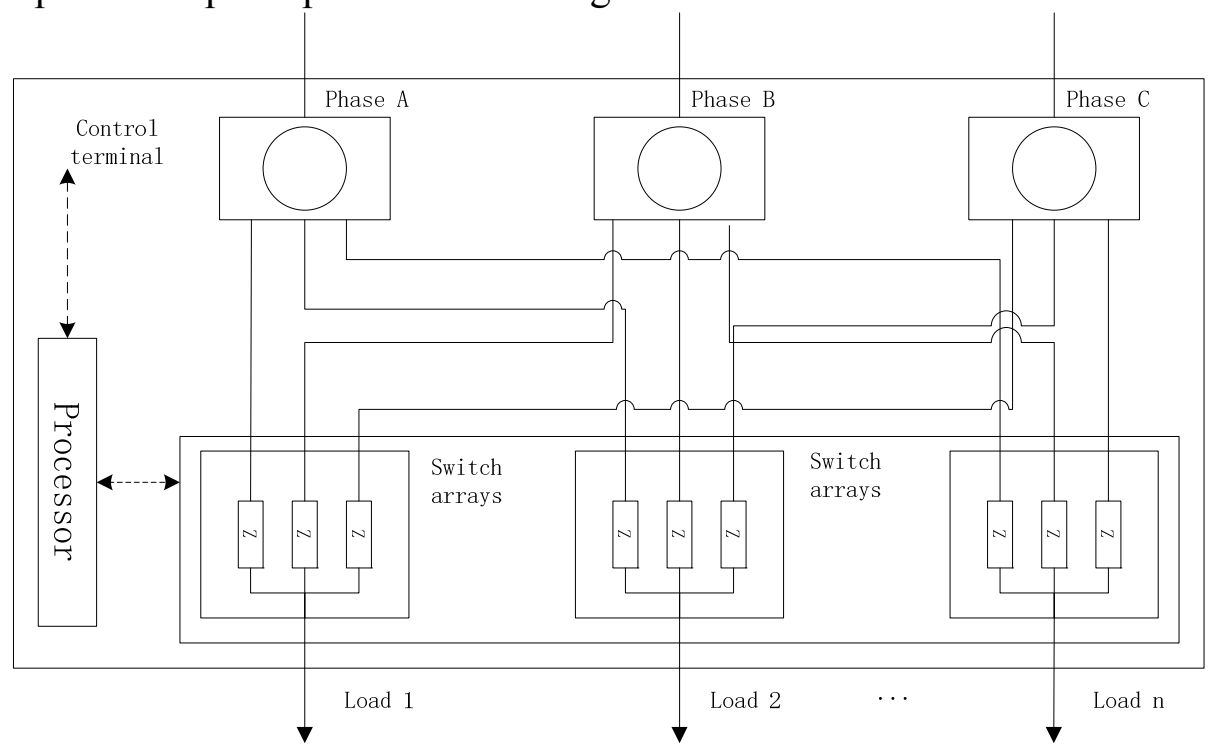

Figure 2 the structure of automatic phase switcher

The master controller of automatic phase switcher used to collect three phase current and neutral line current, according to the set of commutation control strategy, by automatically switching unit to change the phase of power users, improve the degree of three-phase imbalance.

\subsection{Artificial adjustment phase}

The strategy of artificial phase adjustment based on differential evolution optimization calculation, measures the data of imbalance of the transformer and load of single-phase user continuously, monitoring point should be representative, to reflect the user's load characteristics, optimizing decision for the measurement data is calculated, then, adjustment schemes are obtained. When select Single-phase user should investigate the user's characteristics firstly, analysis the location of single-phase users, choose different characteristics of the electricity users, through the phase sequence adjustment between different characteristics of electricity users, improve inconsistent with three-phase load characteristics of the changeable conditions; Phase adjustment mainly concentrated on the load of the maximum and minimum phase sequence.

\section{Example analysis}

Chooses agricultural distribution network area of Hubei province as the research example, the phenomenon of three-phase imbalance is seriously. Tests export current of transformer on a typical day, as shown in table 1. 
Table 1 The data of export current and degree of imbalance

\begin{tabular}{cccccccc}
\hline \multicolumn{3}{c}{ Phase current/A } & \multicolumn{2}{c}{ Degree of phase imbalance/\% } & $\begin{array}{c}\text { Degree of } \\
\text { three-phase } \\
\text { imbalance/\% }\end{array}$ \\
\hline Time & Phase A & Phase B & Phase C & Phase A & Phase B & Phase C & $/$ \\
0:00 & 92.3 & 154.3 & 165.3 & -32.77 & 12.38 & 20.39 & 20.39 \\
$2: 00$ & 120.3 & 194.3 & 181.3 & -27.22 & 17.54 & 9.68 & 17.54 \\
$4: 00$ & 165.3 & 245.2 & 220.3 & -21.39 & 16.61 & 4.77 & 16.61 \\
$6: 00$ & 196.2 & 258.6 & 286.5 & -20.60 & 4.65 & 15.94 & 15.94 \\
$8: 00$ & 220.7 & 314.3 & 298.8 & -20.59 & 13.08 & 7.51 & 13.08 \\
$10: 00$ & 296.2 & 378.6 & 310.7 & -9.83 & 15.25 & -5.42 & 15.25 \\
$12: 00$ & 342.5 & 398.4 & 334.5 & -4.45 & 11.14 & -6.69 & 11.14 \\
$14: 00$ & 315.6 & 388.5 & 316.8 & -7.26 & 14.16 & -6.91 & 14.16 \\
$16: 00$ & 246.3 & 357.6 & 330.1 & -20.89 & 14.86 & 6.03 & 14.86 \\
$18: 00$ & 224.3 & 324.5 & 296.7 & -20.41 & 15.14 & 5.27 & 15.14 \\
$20: 00$ & 162.3 & 298.5 & 289.6 & -35.11 & 19.34 & 15.78 & 19.34 \\
$22: 00$ & 116.5 & 246.2 & 212.6 & -39.25 & 28.39 & 10.86 & 28.39 \\
\hline
\end{tabular}

From the data of above table 1 shows that, average load degree of imbalance reached $16.82 \%$, the average degree of phase imbalance is $-21.65 \%, 21.65 \%$ and $21.65 \%$ respectively. Then statistical process the data of transformer primary side and terminal watt-hour meter, the total loss of station area branch is $198.62 \mathrm{kWh}$, the average power consumption is $8.28 \mathrm{~kW}$.

Installed a compensation device in low voltage side to govern the three-phase imbalance, and then tested current after the compensation, the results were shown in table 2:

Table 2 The test data of imbalance after compensation on a typical day

\begin{tabular}{cccccccc}
\hline \multicolumn{3}{c}{ Phase current/A } & \multicolumn{3}{c}{ Degree of phase imbalance/\% } & $\begin{array}{c}\text { Degree of } \\
\text { three-phase } \\
\text { imbalance/\% }\end{array}$ \\
\hline Time & Phase A & Phase B & Phase C & Phase A & Phase B & Phase C & $/$ \\
$0: 00$ & 146.8 & 139.2 & 127.6 & 6.48 & 0.97 & -7.45 & 6.48 \\
$2: 00$ & 156.8 & 146.2 & 145.6 & 4.86 & -2.23 & -2.63 & 4.86 \\
$4: 00$ & 215.6 & 235.2 & 224.3 & -4.19 & 4.52 & -0.33 & 4.52 \\
$6: 00$ & 243.9 & 224.9 & 259.3 & 0.49 & -7.33 & 6.84 & 6.84 \\
$8: 00$ & 314.2 & 306.2 & 278.6 & 4.85 & 2.18 & -7.03 & 4.85 \\
$10: 00$ & 349.2 & 318.6 & 310.9 & 7.04 & -2.34 & -4.70 & 7.04 \\
$12: 00$ & 362.4 & 358.4 & 334.5 & 3.02 & 1.89 & -4.91 & 3.02 \\
$14: 00$ & 368.5 & 348.5 & 316.8 & 6.94 & 1.13 & -8.07 & 6.94 \\
$16: 00$ & 326.9 & 345.6 & 331.1 & -2.28 & 3.31 & -1.03 & 3.31 \\
$18: 00$ & 298.7 & 319.6 & 297.5 & -2.15 & 4.70 & -2.54 & 4.70 \\
$20: 00$ & 257.9 & 237.2 & 248.3 & 4.08 & -4.28 & 0.20 & 4.08 \\
$22: 00$ & 168.3 & 173.5 & 159.7 & 0.68 & 3.79 & -4.47 & 3.79 \\
\hline
\end{tabular}

From the data of above table 2 shows that, the load of the station area after compensation average imbalance degree is $5.03 \%$, fell by $70.10 \%$ compared with before-compensation, the every phase degree of imbalance was $2.48 \%, 0.52 \%, 3.01 \%$, so the compensation had a good effect. According to the above derived formula, can further calculate rate of line loss reduction and energy saving after three-phase unbalanced governance.

The line loss increased by three-phase imbalance before modification:

$r_{1}=5\left(\beta_{a 1}^{2}+\beta_{b 1}^{2}+\beta_{a 1} \beta_{b 1}\right) / 3=6.1795 \%$

The line loss increased by three-phase imbalance after modification:

$r_{2}=5\left(\beta_{a 2}^{2}+\beta_{b 2}^{2}+\beta_{a 2} \beta_{b 2}\right) / 3=0.1285 \%$

After the three-phase imbalance governance, its energy-saving is:

$\Delta(\Delta E)=\Delta E_{\text {unb }} \frac{r_{1}-r_{2}}{r_{1}+1}=11.32 \mathrm{kWh}$ 
Through calculation, the rate of energy-saving after three-phase imbalance governance was 5.69\%, thus it could be seen, in the area which had serious three-phase imbalance, the unbalanced three-phase governance has good energy saving effect, economic benefit and social benefit.

\section{Conclusion}

In this paper, the energy loss caused by three-phase imbalance which is one of the continuous power quality problems had been quantized, the calculation method was given, and researched the energy-saving after the unbalanced three-phase governance, and proposed the corresponding unbalanced three-phase management scheme, which could provide beneficial reference and guidance for energy-saving transformation and economic benefit calculation of rural power grid.

\section{References}

[1] LIU Bingjiang. The operation of distribution transformer maintenance and troubleshooting skills [J]. Rural Electrician, 2012(6):45-46.

[2] WANG Guomin, LI Yongsi. Adjustment of distribution transformer three-phase load balancing method [J]. Rural Electrician, 2007(11).

[3] YU Yong. Research on reactive power dynamic compensation method for three-phase imbalabce [D]. North China Electric Power University(Baoding),2008.

[4] R. Mienski,R. Pawelek. Shunt compensation for power quality improvement using a STATCOM controller[C]: model-ling and simulation. IEEE Proceedings Generation,Transmission \& Distribution.2004,151(2):274-280.

[5] IL Koteswara Rao. An optimization based algoritiim for shunt active filter under unbalanced and nonsinus-oidal supply voltages[C]. 3rd IEEE Conference on Industrial Electronics and Applications. 2008:1457-1480.

[6] SunD I, Abe S, Shoults R, etal.Calculation of Energy Losses in a Distribution System[J].IEEE Trans on Power Apparatus and Systems,1980,99(4):123-126.

[7] Mayordomo Mohamed Izzeddine, Rafael Aseusi. Load and Voltage Balancing Harmonic Power Flows by means of Static Var Compensator[J]. IEEE Transaction on Power Delivery. 2002, 17(3): $1-3$. 